\title{
A comparative study of safety and efficacy of sitagliptin and glimepiride in patients with type 2 diabetes mellitus
}

\author{
Vinitha Kunder ${ }^{1}$, Shabeer $D^{2}$, Chetan D B ${ }^{*}$ \\ ${ }^{1}$ Department of Pharmacology, Father Muller Medical College, Mangaluru, Karnataka, INDIA. \\ ${ }^{2}$ Department of Pharmacology, Rajarajeswari Medical College and Hospital, Bengaluru, INDIA. \\ ${ }^{3}$ Department of Pharmacology, Vijayanagar Institute of Medical Sciences, Ballari, Karnataka, INDIA. \\ Email: chetandb18@gmail.com
}

$\underline{\text { Abstract }}$

\begin{abstract}
Background: The global prevalence of diabetes mellitus (DM) has risen rapidly in the last three decades. The management of DM involves substantial use of healthcare resources, posing an economic burden on society and patients. Objective: To Compare the Safety and Efficacy of Sitagliptin compared with glimepiride in patients with Type 2 DM with background Metformin therapy. Materials and Methods: This study is conducted in a tertiary care hospital in south India, from March to September 2015. Eligible patients were randomized to receive sitagliptin $100 \mathrm{mg}$ and glimepiride $2 \mathrm{mg}$ once daily as add-on therapy for 12 weeks. Demographic variables were recorded on preformed proforma. Control on diet and regular exercise were advised to all the subjects/patients during study period. HbA1C, FBS, weight, Alanine aminotransferase (ALT), serum urea and serum creatinine measurements were carried out in all the patients at week 0 and then at the end of study at week 12 . The primary endpoint was achievement of target HbA1C upper normal limit at the end of the study. Results: A total of 60 patients were enrolled into the study, with 30 in each group. There were 16 males and 14 females in group A, 18 males and 12 females in group B. A significant reduction of HbA1C and BMI in group A taking sitagliptin was noted as compared to glimepiride group. $(\mathrm{p}<0.05)$. Reduction in FBS was comparable in both the groups $(\mathrm{p}>0.05)$. Side effects in both the groups mostly included hypoglycemia, diarrhoea and vomiting. The frequency of occurrence in both the groups was similar without a statistical difference $(p>0.05)$. Conclusion: Evidence from the present study suggests that sitagliptin is as efficacious as glimepiride, as add-on therapy to metformin, in improving glycemic control and is well tolerated without serious side effects. Sitagliptin showed advantages over glimepiride with lesser risk of hypoglycemia. And it was well tolerated and produced weight loss compared to glimepiride.

Key words: Diabetes Mellitus, Sitagliptin, Glimepiride, HbA1C, BMI,
\end{abstract}

*Address for Correspondence:

Dr Chetan D B, Department of Pharmacology, Vijayanagar Institute of Medical Sciences, Ballari, Karnataka, INDIA.

Email: chetandb18@gmail.com

Received Date: 23/04/2020 Revised Date: 19/06/2020 Accepted Date: 31/07/2020

DOI: https://doi.org/10.26611/10101531

This work is licensed under a Creative Commons Attribution-NonCommercial 4.0 International License. (cc) EY-NC

\begin{tabular}{|l|l|}
\hline \multicolumn{2}{|c|}{ Access this article online } \\
\hline Quick Response Code: & Website: \\
& www.medpulse.in \\
& \\
\hline
\end{tabular}

\section{INTRODUCTION}

Diabetes mellitus (DM) is among the most common chronic diseases in the world, affecting an estimated 422 million adults in $2014 .{ }^{1}$ Sedentary life style, obesity, high BMI, decreased physical activity and increasing longevity result in exponential rise in incidence and prevalence of type $2 \mathrm{DM}$. This high prevalence rate is one of the major factors for economic burden to society as well as patients. Type $2 \mathrm{DM}$ is a major risk factor for developing both micro-vascular (retinopathy, nephropathy and neuropathy) and macro-vascular complications (coronary heart disease, cerebrovascular disease and peripheral vascular disease). ${ }^{2}$ Available treatments focus on reducing hyperglycemia and improving insulin sensitivity. These methods mainly target the primary defects and prevention of complications associated with type $2 \mathrm{DM}$, so they are very attractive and need focus. However, despite the wide array of treatment options available, glycemic control declines over time. ${ }^{3}$ 
Unattainable glycemic control is often a result of ongoing deterioration of beta-cell function. The primary goal of treatment is to target glycemic control by maintaining the $\mathrm{HbA} 1 \mathrm{C}$ level at $6-7 \%$ to decrease the incidence of microvascular and macrovascular complications without predisposing patients to hypoglycemia. ${ }^{4}$ Most of the patients with type $2 \mathrm{DM}$ require more than one antidiabetic agents in combination with or without insulin as monotherapy might leads failure in maintaining of glycemic control and may leads to many complications ${ }^{5}$.Currently available antidiabetic agents work by different mechanisms to lower blood glucose levels. But, each of them has got different pharmacokinetic and pharmacodynamic properties which are the major concerns that limit its usage and dosage titration. ${ }^{4}$ Sitagliptin is an oral, once-daily, potent and highly selective dipeptidyl peptidase-4 (DPP-4) inhibitor approved by the US Food and Drug Administration for use with diet and exercise to improve glycemic control in adult patients with type 2 DM. ${ }^{4}$ Inhibition of DPP-4 activity by sitagliptin enhances fasting and postprandial levels of the intact incretins, glucagon-like peptide-1 (GLP-1) and glucose-dependent insulinotropic polypeptide (GIP). ${ }^{6}$ Regulation of glucose level is maintained by incretins as they play an important role in elevating the insulin release in response to meal and decreasing glucagon secretion is also done by GLP-1. Both of these effects are glucosedependent. ${ }^{7}$ It can be used alone or in combination with metformin or a thiazolidinedione (pioglitazone or rosiglitazone) when treatment with either drug alone provides inadequate glucose control. $0.1 \mathrm{~g}$ once a day is the usual adult dose. A dose of $25-50 \mathrm{mg}$ once daily is recommended for patients with moderate-to-severe renal impairment. ${ }^{4}$ There is no significant data regarding the safety and efficacy of this drug in our population, so this study was conducted to compare the safety and efficacy of sitagliptin as compared to glimepiride in patients inadequately controlled with metformin alone.

\section{MATERIAL AND METHODS}

This study was conducted in a tertiary care hospital in south India, from March to September 2015. After obtaining approval from Institutional Ethics Committee, eligible patients were randomized using a randomization software to receive sitagliptin $100 \mathrm{mg}$ and glimepiride $2 \mathrm{mg}$ once daily as add-on therapy for 12 weeks. Demographic variables of the study population like age, gender, smoking history, hypertension were recorded on preformed proforma. Regular exercise and strict diet control were advised to all the subjects during the study period. HbA1C, FBS, weight (Kg), Alanine aminotransferase (ALT), serum urea and serum creatinine measurements were carried out in all the patients at week 0 and then at the end of study at week 12 . The primary endpoint was achievement of target HbA1C upper limit normal (ULN) at the end of the study. Inclusion criteria:

1. Patients with type $2 \mathrm{DM}$ with poor glycemic control on metformin monotherapy

2. FBS and PPBS values more than $100 \mathrm{mg} / \mathrm{dl}$ and $140 \mathrm{mg} / \mathrm{dl}$ respectively

3. Patients with $\mathrm{HbA} 1 \mathrm{C}$ levels of $>7 \%$

4. Patients of both sexes are included.

\section{Exclusion criteria:}

1. Study subjects with any history of allergy or hypersensitivity to the study drugs

2. Patients with type I DM, pregnancy,

3. Patients with impaired renal and liver functions,

4. Uncontrolled diabetes i.e. $\mathrm{HbA} 1 \mathrm{C}>9 \%$ or fasting blood sugar (FBS) $>300 \mathrm{mg} / \mathrm{dl}$,

5. Uncontrolled hypertension and unstable angina were excluded from the study.

\section{Statistical Analysis}

The data were analyzed using SPSS 17 for windows. Sample size was calculated using PS software by using a power of $80 \%$. Comparative analysis between the two groups were done using Chi-square $\left(\mathrm{x}^{2}\right)$ for categorical variables and student ' $t$ ' test for continuous variables where appropriate. A $\mathrm{p}$ value of $<0.05$ was taken as significant.

\section{RESULTS}

A total of 60 patients were enrolled into the study, with 30 in each group. Mean age in sitagliptin group (A) was 45 years, while that of glimeperide group (B) was 47 years. There was no statistical difference between the groups in terms of age distribution. There were 16 males and 14 females in group A, 18 males and 12 females in group B. Mean BMI between the groups were also matched without any statistical difference.

Table 1

\begin{tabular}{cccc}
\hline & \multicolumn{3}{c}{ Demographic Data } \\
\hline & Sitagliptin & Glimeperide & p Value \\
\hline Age in years(Mean \pm SD) & $45 \pm 4.3$ & $47 \pm 903.2$ & 0.56 \\
Sex(M/F) & $16 / 14$ & $18 / 12$ & 0.76 \\
BMI(Mean \pm SD) & $23 \pm 2.5$ & $22 \pm 2.9$ & 0.64 \\
\hline
\end{tabular}


Table 2

\begin{tabular}{cccccc}
\hline \multicolumn{5}{c}{ Sitagliptin Group } & \multicolumn{2}{c}{ Glimeperide Group } \\
\hline & Baseline & Week 12 & Baseline & Week 12 & p Value \\
\hline HbA1C(\%) & $8.02 \pm 0.56$ & $6.48 \pm 0.23$ & $7.98 \pm 0.60$ & $7.02 \pm 0.30$ & 0.04 \\
FBS & $170 \pm 7.8$ & $120 \pm 5.8$ & $165 \pm 6.6$ & $123 \pm 4.3$ & 0.1 \\
BMI & $27 \pm 2.1$ & $24.1 \pm 1.5$ & $28.0 \pm 2.3$ & $27.03 \pm 1.6$ & 0.02 \\
\hline
\end{tabular}

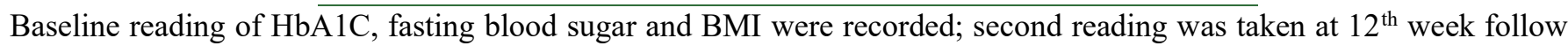
up. Both the readings were compared and analyzed using student $t$ test. There was a statistical difference found in the follow up of $\mathrm{HbA1C}$ and BMI between the Group A and Group B. We found a significant reduction of HbA1C and BMI in group A taking sitagliptin as compared to glimeperide group. $(\mathrm{p}<0.05)$

Reduction in FBS was comparable in both the groups. ( $>0.05)$

Table 3

\begin{tabular}{|c|c|c|c|}
\hline \multicolumn{4}{|c|}{ Side Effect Profile } \\
\hline & Sitagliptin Group & Glimeperide Group & p Value \\
\hline Hypoglycemia & 3 & 2 & 0.56 \\
\hline Diarrhoea & 2 & 1 & 0.98 \\
\hline Vomiting & 2 & 3 & 0.76 \\
\hline Others & 1 & 2 & 0.44 \\
\hline
\end{tabular}

Side effects in both the groups mostly included hypoglycemia, diarrhoea and vomiting. The frequency of occurrence in both the groups was similar without a statistical difference $(p>0.05)$. These side effects were mild and did not need stoppage of medication or resulted in drop outs.

\section{DISCUSSION}

Diabetes mellitus is a major risk factor for developing numerous complications ranging from microvascular injury to organ failure. ${ }^{2}$ The primary objective of the treatment of DM is to maintain the blood glucose levels in the normal range. $\mathrm{HbAlC}$ is a marker of that parameter that reflects the glucose control over past 2 to 3 months. Maintaining $\mathrm{HbA} 1 \mathrm{C}$ at a range of $6-7 \%$ is taken as adequate and reflects a good control of DM. ${ }^{4}$ The American Diabetes Association guidelines state that metformin, along with lifestyle changes, should be considered first-line therapy in patients with type $2 \mathrm{DM}$. If glycemic control not successfully achieved and DM still remains uncontrolled during step-1/first line therapy, then employment of step-2 may be needed which includes sulfonylureas, thiazolinediones or insulin etc. ${ }^{8}$ Metformin and TZDs are the two major drugs in treatment of DM, act by treating the insulin resistance, however they have got no action on declining the progression of beta cell function which observed in patients with type $2 \mathrm{DM}$. So, there is need of newer treatment approaches. Targeting the incretin mimetic hormone is one among them. GLP-1, an incretin hormone, is released when blood glucose levels are elevated, GLP- 1 stimulates insulin secretion, decreases glucagon secretion, improves beta-cell function, and slows gastric emptying. There will be reduction in the production of GLP-1 in patients with type 2 DM. DPP-4 is the enzyme that causes rapid degradation of GLP-1 when it is produced. ${ }^{9}$ So, the action of GLP-1 hormone can be prolonged inhibiting the enzyme with DPP-4 by drugs like sitagliptin. Once the blood glucose level approaches normal, the amounts of insulin released and glucagon suppressed diminishes, thus preventing an "overshoot" and subsequent hypoglycemia which is seen with some other oral hypoglycemic agents. In our study, Sitagliptin group achieved higher reduction in $\mathrm{HbAlC}$ as compared to patients in glimepiride group but the difference was not statistically significant. Similar results were reported by other studies. In study by Arechavaleta et al.. ${ }^{10}$, there were $65 \%$ of patients achieving target $\mathrm{HbA1C}$ of $<7 \%$. Similarly, in a study by Charbonnel et al.. ${ }^{10}$, in patients using sitagliptin, $47 \%$ of them achieved target HbA1C. FBS was reduced in both the groups but the difference between the two groups was not statistically significant. The result was similar to those reported by other studies. In study by Goldstein et al.. ${ }^{13}$, sitagliptin caused $63.9 \mathrm{mg} / \mathrm{dl}$ reduction in FBS. In study by Charbonnel et al.. ${ }^{11}$. FBS was reduced by $50 \mathrm{mg} / \mathrm{dl}$ in the sitagliptin from baseline, whereas it reduced by $42 \mathrm{mg} / \mathrm{dl}$ in glimeperide group. In our study there was a decrease in BMI of patients of both sitagliptin and glimeperide groups, but the reduction in sitagliptin group was statistically significant than the glimeperide group. Similarly in study by Nauck et al.. ${ }^{12}$ there was a significant weight reduction in sitagliptin group as compared to glimepiride group. In our study there were no reported major side effects.

\section{CONCLUSION}

Evidence from the present study suggests that sitagliptin is as efficacious as glimepiride, as add-on therapy to metformin, in improving glycemic control and is well tolerated without serious side effects. 


\section{REFERENCES}

1. World Health Organization. Diabetes. https://www.who.int/news-room/fact-sheets/detail/diabetes (10 April 2020, date last accessed)

2. UK Prospective Diabetes Study (UKPDS) Group. Effect of intensive blood-glucose control with metformin on complications in overweight patients with type 2 diabetes (UKPDS 34). Lancet 1998;352:854-65.

3. Turner RC, Cull CA, Frighi V, Holman RR. Glycemic control with diet, sulfonylurea, metformin, or insulin in patients with type 2 diabetes mellitus: Progressive requirement for multiple therapies (UKPDS 49). UK Prospective Diabetes Study (UKPDS) Group. JAMA 1999;281:2005-12.

4. Choy M, Lam S. Sitagliptin: a novel drug for the treatment of type 2 diabetes. Cardiol Rev 2007 ; 15:264-71.

5. Inzucchi SE: Oral antihyperglycemic therapy for type 2 diabetes: scientific review. JAMA 2002; 287:360-72.

6. Herman GA, Stevens C, Van Dyck K, Bergman A, Yi B, De Smet $\mathrm{M}$, et al... Pharmacokinetics and pharmacodynamics of single doses of sitagliptin, an inhibitor of dipeptidyl peptidaseIV, in healthy subjects. Clin Pharm Therap 2005;78:675-88.

7. Drucker DJ, Nauck MA. GLP-1R agonists (incretinmimetics) and DPP-4 inhibitors (incretin enhancers) for the treatment of type 2 diabetes. Lancet 2006; 368:1696-1705.

8. Nathan DM, Buse JB, Davidson MB, Heine RJ, Holman RR, Sherwin $\mathrm{R}$, et al... Management of hyperglycemia in type 2 diabetes: A consensus algorithm for the initiation and adjustment of therapy: A consensus statement from the
American Diabetes Association and the European Association for the Study of Diabetes. Diabetes Care 2006;49:2816-8.

9. Nissen SE, Wolski K. Effect of rosiglitazone on the risk of myocardial infarction and death from cardiovascular causes. $\mathrm{N}$ Engl J Med 2007;356:2457-71.

10. Arechavaleta R, Seck T, Chen Y, Krobot KJ, O'Neill EA, Duran L, et al... Efficacy and safety of treatment with sitagliptin or glimepiride in patients with type 2 diabetes inadequately controlled on metformin monotherapy: a randomized, double-blind, non-inferiority trial. Diabetes ObesMetab 2011;13:160-8.

11. Charbonnel B, Karasik A, Liu J, Wu M, Meininger G; Sitagliptin Study 020 Group. Efficacy and safety of the dipeptidyl peptidase-4 inhibitor sitagliptin added to ongoing metformin therapy in patients with type 2 diabetes inadequately controlled with metformin alone. Diabetes Care 2006;29:2638-43.

12. Nauck MA, Meininger G, Sheng D, Terranella L, Stein PP; Sitagliptin Study 024 Group. Efficacy and safety of the dipeptidyl peptidase-4 inhibitor, sitagliptin, compared with the sulfonylurea, glipizide, in patients with type 2 diabetes inadequately controlled on metformin alone: a randomized, double-blind, non-inferiority trial. Diabetes ObesMetab 2007;9:194-205.

13. Goldstein BJ, Feinglos MN, Lunceford JK, Johnson J, Williams-Herman DE; Sitagliptin 036 Study Group. Effect of initial combination therapy with sitagliptin, a dipeptidyl peptidase-4 inhibitor, and metformin on glycemic control in patients with type 2 diabetes. Diabetes Care 2007;30:1979-87.

\section{Source of Support: None Declared \\ Conflict of Interest: None Declared}

Policy for Articles with Open Access:

Authors who publish with MedPulse International Journal of Pharmacology (Print ISSN: 2550-7567) (Online ISSN: 2636-4670) agree to the following terms: Authors retain copyright and grant the journal right of first publication with the work simultaneously licensed under a Creative Commons Attribution License that allows others to share the work with an acknowledgement of the work's authorship and initial publication in this journal.

Authors are permitted and encouraged to post links to their work online (e.g., in institutional repositories or on their website) prior to and during the submission process, as it can lead to productive exchanges, as well as earlier and greater citation of published work. 